\title{
La Dynamique de la Fecondite des Adolescentes au Senegal
}

\author{
Nafissatou Jocelyne DIOP
}

\section{I-INTRODUCTION}

L'étude de la fécondité des adolescentes est en train de devenir une sous-discipline principale des études de population (Westoff et al., 1983). En Afrique, cette problématique ne fait que commencer à soulever l'intérêt. Dans plusieurs pays, elle est encore reçue avec beaucoup de scepticisme car elle n'est pas encore perçue comme un problème. Pourtant si l'on se place dans une perspective de diminution de la fécondité, l'apport des naissances des adolescentes est considérable, puisque dans la plupart des pays africains les naissances d'adolescentes comptent pour $20 \%$ des naissances totales (United Nations, 1989). Les cas de grossesses avant le mariage deviennent également de plus en plus importantes (Nichols et al., 1986; Gyepi-Garbrah, 1985) avec des conséquences souvent dramatiques. En effet, le nombre des avortements illégaux a considérablement augmenté avec des conséquences néfastes sur la santé ; des abandons scolaires quand la grossesse survient chez les adolescentes en scolarité ; une augmentation de l'infanticide ; et des répercussions sur la santé des enfants (Omu, 198I; Senderowitz et Paxman, 1986; Akuffo, 1987; Hobcraft, 1987; Adetoro et Agah, 1988).

Ainsi grâce à la littérature plusieurs aspects pertinents pour une recherche sur la fécondité des adolescentes en Afrique ont été identifiés. Parmi ces aspects figurent en bonne place la préoccupation pour l'activité sexuelle des jeunes filles célibataires, urbaines et en scolarité. Cependant, les explications avancées comme déterminantes de cette activité sexuelle sont tirées de contextes d'études trop restrictifs (Ladipo et al., 1983; Akuffo, 1987; GyepiGarbrah, 1985; Nichols et al., 1986).

La fécondité des adolescentes mariées a quant à elle fait l'objet de très peu d'études, ce qui, dans les pays développés se justifie amplement car plus de $90 \%$ des adolescentes y sont célibataires. Mais, dans les pays africains, cette situation est simplement inadmissible. En effet dans ces pays, c'est plus de la moitié des adolescentes qui sont mariées. De surcroît, elles vivent dans les milieux ruraux et n'ont aucune instruction. En adoptant ce cadre d'étude restrictif, seule une minorité d'adolescentes seront concernées alors que c'est la grande majorité de ces femmes qui sont intéressées car ce sont elles qui ont besoin d'un programme d'intervention.

L'objet de cette étude est d'analyser la fécondité durant l'adolescence d'une manière plus générale, afin de mieux percevoir les changements qui ont eu lieu. Cette étude part de l'hypothèse de base que les changements socio-économiques importants intervenus dans les sociétés africaines durant les dernières décennies, ont perturbé les trajectoires de vie des adolescentes.

Dans la plupart des dictionnaires, l'adolescence est définie comme "le processus à travers lequel un individu fait la transition du stade de l'enfance à celui d'adulte". L'adolescence se caractérise par des efforts pour atteindre "un développement social, physique, mental et émotionnel" (Elder, 1980). Si, dans la plupart des cultures, c'est le début de la puberté qui marque le début de l'adolescence, la fin de l'adolescence n'est pas, quant à elle, clairement définie (Senderowitz et Paxman, 1985; WHO, 1986). Senderowitz et Paxman (1985) pensent que la définition de l'adolescence peut varier d'une culture à l'autre et tant que certains critères reconnus comme ceux d'un adulte ne se sont pas manifestés, on ne pourra pas parler d'adolescent dans cette culture. Aussi ce qui définit l'adolescence n'est pas la puberté, ou la croissance soudaine, ou le processus de maturation générale, mais plutôt la perception de cette maturation communement admise dans la société considérée. Or le mariage est synonyme de respectabilité, de maturité et de statut d'adulte. La maternité également est reconnue comme telle, et même quand elle arrive en dehors du mariage, l'adolescente perd tous ses privilèges de jeune fille, dont la prise en charge 
matérielle et sociale par les parents. Ainsi dans la société sénégalaise, la fin de l'adolescence est marquée par le mariage ou la maternité. Pour les besoins de l'analyse, nous avons adopté la définition de travail suivante : "tout événement survenu avant l'âge de vingt ans marque la fin de l'adolescence". Et cela pour toutes les générations de l'enquête.

Les déterminants proches qui permettent d'expliquer la fécondité des adolescentes sont au nombre de quatre:I) La puberté qui marque biologiquement le début d'exposition au risque de grossesse; 2 ) Le mariage qui marque socialement le début de l'exposition au risque de grossesse; 3 ) La contraception dont l'utilisation empêche la grossesse; 4) Et l'avortement provoqué qui met fin à une grossesse non-désirée.

Dans cet article nous ne retenons que deux déterminants proches : la puberté et le mariage.

\section{II-SOURCE DES DONNÉES MÉTHODOLOGIE}

\section{a- Source des données et limites de l'étude}

Cette étude utilise l'Enquête démographique et de santé du Sénégal (EDS) qui a été réalisée par la Direction de la Statistique et des Enquêtes Démographiques d'avril à août 1986. L'enquête individuelle a porté sur 4415 femmes âgées de 15 à 49 ans. Nous allons mener une analyse longitudinale à partir des trois événements que sont la puberté, le mariage, la première maternité. Cette approche longitudinale permet de faire ressortir les effets de génération et donc montre l'évolution des événements avant l'âge de 20 ans pour les générations de femmes de 15 à 44 ans. L'inconvénient majeur de ce type d'analyse réside dans les erreurs de déclarations aux questions rétrospectives, surtout lorsque les événements sont très anciens. Pour cette raison, nous avons préféré éliminer le groupe d'âge 45-49 ans. Cependant, la fécondité des adolescentes n'étant qu'un aspect particulier de la fécondité féminine en général, elle reste très influencée par les caractéristiques collectives liées au milieu socioculturel et contextuel.

\section{b. Méthode d'analyse}

La méthode la mieux adaptée à ce type d'étude utilise les tables de survie. En effet celles-ci permettent d'éviter des erreurs systématiques, d'utiliser toutes les données et de tirer parti de l'information des histoires incomplètes (Le Bourdais et Desrosiers, 1990).

Dans une enquête transversale comme celle de I'EDS, l'expérience de certaines femmes de l'échantillon ou leur exposition à un risque sont interrompues par l'enquête car les événements ultérieurs qu'elles vivront ne seront pas pris en compte. Par exemple, les histoires des générations de 15-19 ans et de 20-24 ans sont incomplètes car un nombre important de femmes n'auront pas encore vécu l'événement étudié: ce sont les cas tronqués. II existe une hypothèse fondamentale qui s'applique à toute table d'éventualité: c'est l'hypothèse markovienne où l'on suppose que la probabilité de transition d'un individu ne dépend que de l'état où il se trouve au début de chaque période de transition (Aumont, 1987). Etant donné la spécificité de cette étude, seuls les phénomènes de rang $I$ sont pris en compte : le premier mariage et la première naissance.

Pour la plupart des tables où l'on compare les générations, nous utilisons des tables brutes qui décrivent l'apparition d'événements en l'absence de phénomènes perturbateurs et permettent la description d'un phénomène à l'état pur (Smith, 1980). Ainsi une table nette fondée sur deux phénomènes concurrents est construite en considérant deux causes d'éventualité: les conceptions prénuptiales et les conceptions après le mariage.

Nous utilisons également des tests d'égalité des courbes de survie et des tests d'associations des variables. Quand on mène une analyse comparative entre plusieurs courbes de survie, il est utile d'avoir recours à des tests pour synthétiser les différences entre des fonctions de survie sur toute la période de l'étude (Kalbfleisch et Prentice, 1980). La seule manière de tester les distributions des tables d'éventualité est d'utiliser les tests de rangs à scores exponentiels. Ces tests sont destinés à comparer le calendrier de plusieurs tables d'éventualité ayant la même allure. 


\section{III-RÉSULTATS}

\section{a- La puberté}

Dans l'EDS, il a été demandé à l'enquêtée "l'âge atteint au moment de l'apparition des menstruations". La qualité de cette variable est difficile à mesurer, car nous n'avons pas de données comparatives. Mais nous estimons que l'apparition des menstruations étant un événement marquant pour la jeune fille, les risques de déplacement ne sont pas plus importants que ceux liés aux déclarations des dates de mariage ou de la première maternité.

Les tables de survie portant les données du Sénégal montrent qu'il n'y a pas eu de changements majeurs pour les générations nées entre 1940 et 197I, comme l'indique l'allure générale des courbes (figure I). Cependant les générations nées entre 1966 et I97/ ont eu leurs menstruations légèrement plus tôt que les générations nées entre 1951 et 1956, entre les âges de 14 et 16 ans. Mais la différence reste très faible et n'a pas l'ampleur de la baisse constatée en France qui a été de 2 ans et 4 mois en 46 ans, ou celle constatée aux Etats-Unis, un an par génération.

A 18 ans, l'âge auquel toutes les femmes doivent être pubères, aucune tendance ne se dégage. L'âge médian cependant laisse apparaître une baisse à peine sensible dans la dernière génération (Tableau I). II faudrait cependant mener l'observation sur une période plus longue pour confirmer ou infirmer la tendance à la baisse de la puberté, bien que l'Enquête Sénégalaise sur la Fécondité de 1978 ait donné les mêmes résultats.

Un test de rangs à scores exponentiels mené sur l'association entre l'âge à la puberté et les deux variables explicatives que sont le lieu de résidence et le niveau d'éducation a révélé qu'aucune des deux variables n'est en réalité significative quand tous les groupes d'âge sont considérés. L'instruction et le lieu de résidence ne déterminent donc pas le calendrier de l'âge à la puberté quand on prend en considération toutes les générations. Les conditions de vie, en particulier le niveau de nutrition dans le milieu urbain et le milieu rural, peuvent avoir été longtemps les mêmes, avec quelquefois de meilleures conditions dans les campagnes, qui sont les principales sources de production alimentaire. La nourriture est plus facile à trouver, et à des prix plus abordables. Cependant avec la sécheresse qui a commencé vers le milieu des années 1970 au Sahel et la crise économique, l'équilibre alimentaire des campagnes s'est complètement bouleversé. Les conditions de vie ont commencé à être nettement meilleures dans les villes.

Relation entre la puberté, le premier mariage et la première naissance

Le calendrier du mariage selon l'âge auquel les femmes ont eu leur puberté a montré que quand l'âge à la puberté est précoce, l'âge au mariage l'est également, l'intervalle entre la puberté et le mariage étant très court. Ainsi, parmi les femmes qui ont eu leurs menstruations à 13 ans, $83 \%$ étaient déjà mariées à 18 ans, alors que parmi celles qui ont eu leurs menstruations à 16 ans, 61 $\%$ l'étaient à 18 ans également (figure 2).

Le tableau 2 montre aussi qu'un nombre non négligeable de femmes sont mariées avant l'apparition de leur puberté. Si culturellement cela se conçoit dans certaines ethnies comme chez les Poulars et les Soninké où les filles sont promises en mariage à une autre famille dès l'enfance, nous ne devrions pas les voir apparaître ici, car avant la puberté, l'union n'est en principe jamais consommée. Or l'objectif de l'EDS était de déterminer l'âge à la consommation du mariage afin de cibler les femmes soumises au risque de conception. C'est pourquoi théoriquement l'âge au mariage que nous utilisons est en réalité l'âge à la consommation du mariage. Nous pensons pouvoir ainsi éviter les confusions qui pourraient provenir du caractère spécifique du mariage traditionnel au Sénégal.

La relation entre les menstruations et la maternité qui vient d'établir que les femmes qui ont eu leur menstruation précocément ont eu également une première naissance précoce, est manifeste pour les âges aux menstruations de 14, 15 et 16 ans. Mais cette relation est encore plus apparente aux jeunes âges, et s'atténue au fur et à mesure que l'âge augmente. Ainsi à l'âge de 23 ans, la proportion des femmes qui ont déjà eu un enfant est pratiquement la même quel que soit l'âge 
auquel la puberté est survenue (figure 3). Cette relation mérite d'être soulignée car elle confirme que dans des populations non malthusiennes, le début de la fécondité est déterminé également par l'âge à la puberté.

Cependant, entre les générations nées entre 1946 et I97I, la baisse de l'âge aux menstruations n'est pas aussi manifeste. II faudrait une observation plus longue et des données plus fiables pour pouvoir tirer des conclusions plus nettes. L'apparition des menstruations chez une adolescente est également interprétée comme un signe indiquant que l'adolescente peut être donnée en mariage et consommer ce mariage. En effet la relation qui a été établie entre l'âge à la puberté et l'âge au mariage a montré que dans la société, l'apparition des menstruations signifie que la fille est physiquement mature pour la reproduction et donc pour le mariage. La puberté est donc un déterminant non négligeable pour la formation des familles. La procréation suit très vite.

\section{b. L'entrée en union}

L'exposition au risque de concevoir est déterminée aussi bien par des facteurs physiologiques comme la puberté, que par des facteurs de comportement. Le premier facteur nécessaire pour qu'il y ait exposition au risque de grossesse se trouve être l'existence préalable de rapports sexuels. Ceux-ci peuvent avoir lieu dans le mariage ou en dehors de tout lien matrimonial. En particulier dans une société islamisée avec des normes sociales traditionnelles encore très fortes comme celles du Sénégal, le mariage demeure le seul cadre socialement admis pour les relations sexuelles. La plupart des études faites sur la fécondité reconnaissent que le mariage sanctionne l'entrée dans la vie féconde.

II ressort de l'analyse bivariée que $17,4 \%$ de l'ensemble des femmes se sont mariées avant leur quinzième anniversaire, mais que le plus grand nombre de mariage $(43,6 \%)$ intervient entre 15 et 17 ans (Rapport EDS-Sénégal, 1988). Au total c'est près de $89 \%$ des femmes mariées qui l'ont été avant 20 ans, ce qui confirme la très forte intensité du mariage durant la période de l'adolescence.
Les données du tableau 2 font apparaître une modification de la nuptialité. En effet il y a eu une élévation de l'âge au premier mariage. Pour un âge donné d'entrée en union, les proportions cumulées de non mariées sont d'autant plus fortes entre les différentes générations que la génération est jeune. Les jeunes générations sont donc entrées en union plus tardivement que leurs aînées ou leurs mères.

Si l'on considère les mariages qui ont été conclus avant 15 ans, donc à un âge très précoce, au I5ième anniversaire, $20 \%$ des femmes âgées de $40-44$ ans, $17 \%$ des femmes de $25-29$ ans et 13 $\%$ des femmes de 15-19 ans étaient déjà mariées. On est donc en présence d'une régression régulière des mariages précoces des vieilles générations aux générations les plus jeunes. Les mutations les plus importantes se sont produites chez les générations qui sont nées entre 1966 et 197I (figure 4). En effet l'écart d'entrée en union entre les générations âgées de 15-19 ans et 20-24 ans est assez important pour conclure que l'âge au mariage a régulièrement baissé depuis 20 ans, mais qu'il $y$ a eu une accélération ces dernières années. Cependant une partie de cette baisse est surestimée puisqu'il y a eu une sous-estimation des femmes mariées dans le groupe d'âge 15-19 ans, selon le rapport d'évaluation des Enquêtes Démographiques et de Santé.

Si les proportions de célibataires sont de plus en plus fortes à mesure que la génération est jeune, la tendance à la hausse que nous avons remarquée de l'âge au premier mariage n'est pas très nette dans tous les groupes d'âge. On peut également voir au tableau 2 que le nombre de femmes qui se marient entre 15 et 19 ans a régulièrement baissé, passant de $61 \%$ pour les $40-44$ ans à $38 \%$ pour les 15-19 ans. Au total à 19 ans, c'est une femme sur 5 qui est encore célibataire dans le groupe d'âge 40-44 ans et c'est une femme sur 2 qui est encore célibataire dans le groupe d'âge 15-19 ans. Et ce sont surtout les mariages conclus entre 15 et 17 ans qui ont baissé de moitié.

La proportion de femmes mariées avant 20 ans a donc régulièrement diminué au fil du temps. Le test du $\mathrm{khi}^{2}$ se révèle significatif au seuil de $\mathrm{I} \%$.

Etant donné que le mariage marque socialement la fin de l'adolescence, l'adolescence s'est donc 
progressivement allongée. Si quatre-cinquième de la génération née en 194I-1947 n'étaient plus adolescentes avant d'atteindre le vingtième anniversaire, c'est seulement la moitié de la génération née en 1966-197| qui ne l'est plus avant d'atteindre le vingtième anniversaire. Pour mieux expliquer les variables associées au temps de sortie du célibat, nous avons retenu 5 variables qui pourraient avoir une influence: la résidence et l'éducation, l'âge à la puberté, et la survie de la mère et du père au moment du mariage. Ces deux dernières variables sont pertinentes dans la mesure où le mariage est une affaire de famille. En outre, l'autorité des parents étant prééminente en Afrique, ces variables nous permettent de mieux appréhender le contrôle familial.

Ces variables sont toutes significatives dans le test univarié de Wilcoxon où elles sont examinées une à une, toute chose égale par ailleurs. Selon le test univarié du Log rank, l'âge à la puberté n'est pas significatif. Dans la réalité, il est difficile de chercher à isoler les variables puisqu'il existe des interactions entre elles. Dans les deux cas, c'est l'instruction qui est la variable la plus fortement associée à l'âge d'entrée en union, suivie par la résidence. L'âge à la puberté vient en 3ème position. Par contre si la survie du père au moment du mariage est significative, celle de la mère ne semble pas jouer un rôle important dans le retard ou la précocité de l'âge au mariage.

Ainsi le Sénégal garde encore un profil de société où le mariage est précoce. Si l'on a pu assister à une hausse régulière de l'âge d'entrée en union depuis 25 ans, celle-ci s'est accélérée avec les générations nées en 1966. II y a eu moins de mariages entre 15 et 17 ans pour ces générations alors que les mariages avant 15 ans sont restés presque constants.

Socialement la période d'adolescence s'est allongée avec la jeune génération. II semble que c'est l'instruction qui a joué un rôle prédominant dans cet allongement de l'adolescence, puisque plus les jeunes filles sont instruites moins leur sortie de l'adolescence est précoce. Une instruction même légère produit un allongement de la période d'adolescence. Cela est dû au fait que les parents qui envoient leurs filles à l'école sont plus soumis à l'influence de l'occident à travers les mass-médias, les ouvrages scolaires et l'enseignement, qui véhiculent plus de liberté de l'individu. Ces changements culturels peuvent se traduire par une augmentation de la préférence et de la tolérance du libre choix d'un mari (Lesthaege et al., 1989; Caldwell, 1990), ce qui se fait toujours plus tard que lorsque le mari est imposé par la famille. Cependant même quand le choix est fait individuellement, le contrôle et l'influence de la famille ont complétement disparu.

L'âge à la puberté est donc intimement lié à la durée de l'adolescence. Ainsi une jeune fille qui a ses menstruations précocement est mariée également très tôt. Mais les rapports entre la puberté et les autres facteurs ne sont pas aussi contraignants.

Par contre la survie du père est positivement reliée à l'âge au mariage, contrairement à I'hypothèse qui avait été faite. La seule explication que nous pouvons trouver est que le père de famille étant le soutien financier de la famille, son décès peut entraîner le mariage précoce de la jeune fille, puisque le nouveau mari apportera ainsi un nouveau soutien matériel à toute la famille. Cette explication s'insère dans une stratégie de survie de la famille pour maintenir son niveau de vie. Par contre la présence de la mère n'a pratiquement aucun effet sur la durée de l'adolescence.

Les résultats obtenus avec l'EDS montrent donc que le début de l'adolescence se fait en moyenne autour de l'âge de 15 ans, et cela n'a pas changé depuis 6 générations au Sénégal. La période d'adolescence est par conséquent assez courte, puisque nous avons vu que la sortie de l'adolescence, qui est marquée par le mariage, se fait en moyenne autour de 17 ans, donc deux ans après la puberté pour l'ensemble des femmes. Cependant la fin de l'adolescence se fait plus tard avec les plus jeunes générations.

Le schéma de l'augmentation du décalage "biosocial", nous a montré que désormais, au Sénégal, le fossé s'agrandit entre la période de début et celle de la fin de l'adolescence, ce qui allonge la période sociale d'adolescence. Cet allongement n'est pas dû à des changements dans la maturité biologique, mais dans la maturité sociale.

\section{c. La fécondité avant $\mathbf{2 0}$ ans}


Pour mettre en évidence l'évolution de l'âge à la maternité, nous avons fait une table de survie d'occurrence de la première naissance par génération qui permet de mieux apprécier ce que pourrait être le changement dans la jeune génération qui n'a pas eu le temps de vivre toute la période qui précède le 20 ème anniversaire. La figure 6 montre que seule la courbe des 15-19 ans se détache réellement des autres. Les courbes des autres générations sont confondues (figure 6), mais le test du Log-rank nous montre qu'elles sont significativement différentes (seuil de 1\%). Le test de comparaison entre deux proportions n'est significatif que entre les groupes $15-19$ ans et 2024 ans. En effet, au I8ème anniversaire, pour les générations de 20 à 44 ans, le pourcentage des femmes qui ont eu une naissance varie entre 39 et $40 \%$, alors qu'il est de $31 \%$ pour les I5- I 9 ans. II semble donc que c'est avec la génération la plus jeune que l'on assiste à une baisse de la fécondité avant 20 ans (Tableau 4).

Les naissances très précoces survenues avant 15 ans sont relativement rares. A 15 ans, pour les générations de 20 à 44 ans, il y a eu environ $5 \%$ de femmes qui ont eu une naissance. Pour le groupe d'âge 15-19 ans ce pourcentage est d'environ $3 \%$. Il y a donc eu une légère baisse des naissances avant I 5 ans.

L'âge de la première naissance n'a donc que légèrement augmenté au fil du temps, le gain ayant été exclusivement apporté par la jeune génération. La fécondité est restée constante pendant trois générations, puis elle a légèrement augmenté avec les générations nées entre $196 \mathrm{I}$ et 1966, et n'a commencé à baisser qu'avec les générations nées après 1966. Et pourtant nous avons vu que l'âge d'entrée en union a subi une augmentation graduelle avec les générations : d'où la conclusion paradoxale que malgré l'augmentation graduelle de l'âge au premier mariage, la fécondité n'évolue pas d'une manière significative. Différents arguments peuvent être avancées pour expliquer ce constat :

- Les femmes les plus âgées ont déclaré les dates de naissance de leur premier enfant plus tard qu'elles n'ont eu lieu, cela conformément à la tendance des femmes de ce groupe d'âge à omettre la première naissance ou à la déclarer comme plus tardive qu'elle ne l'a réellement été
(Trussell et Reinis, 1990). Dans ce cadre, il faut rappeler que la déclaration de l'âge auquel sont survenus les événements et la déclaration des événements eux-mêmes sont des problèmes existant dans toutes les enquêtes africaines. Cependant des efforts ont été faits dans l'EDS pour que les déclarations de dates soient le plus près possible de la réalité. D'autre part, les femmes de moins de 30 ans ont été identifiées comme ayant les meilleurs enregistrements (Mboup, 1992). Or c'est chez ces trois jeunes générations qu'on a constaté une hausse de l'âge au mariage. Si la fécondité avant 20 ans avait réellement subi une baisse régulière, on devrait la voir apparaître avec ces trois générations.

- La mortalité intra-utérine a pu baisser suite à l'amélioration des conditions sanitaires (Ndiaye et al., 1988).

- Il peut y avoir eu une augmentation de la fécondité avant le mariage.

- Il peut y avoir eu un raccourcissement de l'intervalle protogénésique lorsque l'âge au premier mariage augmente.

- II peut y avoir une infécondité chez les adolescentes.

\section{Les conceptions prénuptiales}

Les conceptions prénuptiales comprennent toutes les naissances qui ont eu lieu avant sept mois révolus après le mariage. Cette durée de sept mois a été retenue afin d'éliminer les naissances prématurées qui pourraient avoir lieu. En effet, la limite entre conceptions prénuptiales et post-nuptiales fondée sur des durées de mariage lors de la naissance, se définit difficilement en raison de la variabilité de la durée de grossesse. Selon Pressat (1983), on peut néanmoins opérer une séparation statistiquement satisfaisante. Ainsi à 8 mois exacts de mariage, toutes les naissances intervenues de 0 à 7 mois révolus seront considérées comme résultant de conceptions prénuptiales, celles étant intervenues au-delà étant attribuées à des conceptions postnuptiales. Toujours selon Pressat, ce mode de partage repose sur le fait qu'il y a sensiblement compensation entre les naissances relevant de conceptions prénuptiales et ayant lieu au delà de huit mois et celles relevant de conceptions postnuptiales et ayant lieu avant 8 mois. Au Sénégal, les naissances avant le mariage et les conceptions 
prénuptiales représentent $29,8 \%$ du total des premières naissances, alors qu'en ne considérant que les naissances prénuptiales, ce pourcentage est de 17,3\% (Tableau 5).

Pour mieux apprécier comment les femmes sénégalaises entrent en maternité, et afin de remettre cet événement dans une perspective de cycle de vie, nous utilisons une table de survie multidimensionnelle, qui dans ce cas-çi est composée de deux événements concurrents. Ce type de table rendra mieux compte des transitions effectuées de l'état de jeune fille sans enfant à celui de mère, en prenant en considération le statut matrimonial au moment où l'événement se produit. Ainsi le premier événement est constitué des conceptions et des naissances qui ont eu lieu avant le mariage, et le deuxième événement des mariages ayant eu lieu avant les conceptions. Pour les besoins de l'élaboration de la table nous avons regroupé les naissances prénuptiales et les conceptions prénuptiales. C'est pourquoi, pour le reste de l'analyse nous avons privilégié le terme de "conceptions" prénuptiales.

L'examen des tables nous montre que les proportions cumulées de conception hors mariage avant 16 ans ont fortement baissé, alors qu'elles ont presque stagné avant 18 ans. La légère baisse de ces proportions cumulées avant 18 ans avec le groupe d'âge $15-19$ ans doit être interprétée avec beaucoup de prudence car ce groupe d'âge vit encore l'événement (Tableau 6).

Par contre lorsque l'on regarde ce que représente en pourcentage ces proportions cumulées de conceptions prenuptiales par rapport aux femmes qui ont vécu un événement, on constate une différence de comportement des deux plus jeunes générations. Le pourcentage de femmes qui ont commencé leur vie adulte par une conception prénuptiale a augmenté de $6 \%$ en valeur absolue entre les 20-24 ans et les 25-29 ans, et de $3 \%$ entre les 15-19 ans et les 20-24 ans. Pour les quatre groupes les plus âgés, il y a eu une relative stabilité de ces pourcentages et nous mettons les légères fluctuations sur le compte des erreurs de déclaration (tableau 6).

Ainsi cette enquête n'a pas confirmé l'hypothèse de l'augmentation des conceptions prénuptiales. Donc nous ne trouvons pas ici l'explication de la faible évolution de la fécondité avant 20 ans, en dépit de la modification de nuptialité.

Par contre cette étude des conceptions prénuptiales avant l'âge de 19 ans par génération nous a permis de voir qu'un changement a été amorcé avec les générations nées à partir de 1961, puisque ces femmes ont été plus nombreuses à connaître comme premier événement une conception prénuptiale (Tableau 6).

Autrement dit, lorsque la période d'adolescence s'allonge, avec l'augmentation de l'âge au mariage, il se crée un décalage bio-social. Ce décalage entre la maturité biologique et la maturité sociale entraîne l'augmentation du nombre des femmes ayant connues une conception prénuptiale en premier événement. En effet, nous avons vu que c'est pour les deux générations les plus jeunes que l'allongement de la période d'adolescence est la plus importante, et ce sont ces générations qui ont vécu un plus grand nombre de conceptions avant le mariage comme premier événement. Cependant c'est surtout pour la dernière génération que le changement de comportement est le plus frappant. Mais étant donné que ce groupe n'a pas fini de vivre l'événement, son comportement actuel n'est certainement pas définitif.

\section{Implications pour la politique de population}

De cette recherche on peut tirer plusieurs implications:

I) Le mariage n'est pas le début de l'exposition au risque de grossesse, comme l'ont fait croire la plupart des études sur la fécondité. En effet nous avons vu qu'un pourcentage non négligeable de femmes ont connu une conception prénuptiale comme premier événement.

2) Un décalage est en train de se produire entre la maturité sexuelle et la maturité sociale, provoquant un allongement de la période d'adolescence. Cet allongement de la durée de l'adolescence a pour effet majeur d'augmenter le nombre de femmes qui connaissent une naissance ou une conception avant le mariage comme premier événement, créant ainsi des drames familiaux et sociaux. 
C'est pourquoi il devient urgent de mettre en place des mesures d'accompagnement pour pallier aux conséquences souvent dramatiques de cet allongement de l'adolescence.
Particulièrement en mettant en place une politique de planification familiale spécifique aux jeunes.

Tableau I: Proportion des femmes non pubères par génération

\begin{tabular}{|c|c|c|c|c|c|c|}
\hline Age à l'enquête & $15-19$ & $20-24$ & $25-29$ & $30-34$ & $35-39$ & $40-44$ \\
\hline $\begin{array}{l}\text { Année de } \\
\text { naissance }\end{array}$ & $\begin{array}{l}1966- \\
71\end{array}$ & $\begin{array}{l}1961- \\
66\end{array}$ & $\begin{array}{l}1956- \\
61\end{array}$ & $\begin{array}{l}1951- \\
56\end{array}$ & $\begin{array}{l}1946- \\
51\end{array}$ & $\begin{array}{l}1941- \\
46\end{array}$ \\
\hline \multicolumn{7}{|l|}{$\begin{array}{l}\text { Age au début } \\
\text { de l'intervalle }\end{array}$} \\
\hline II & 0,9949 & 0,9955 & 0,9857 & 0,9878 & 0,9896 & 0,9767 \\
\hline 12 & 0,9877 & 0,9944 & 0,9749 & 0,9848 & 0,9833 & 0,9667 \\
\hline 13 & 0,9477 & 0,9542 & 0,9391 & 0,9436 & 0,9563 & 0,9333 \\
\hline 14 & 0,7959 & 0,8302 & 0,8019 & 0,8125 & 0,7979 & 0,8200 \\
\hline 15 & 0,5313 & 0,5888 & 0,5847 & 0,5915 & 0,5750 & 0,6167 \\
\hline 16 & 0,2031 & 0,2145 & 0,2005 & 0,2226 & 0,1729 & 0,2367 \\
\hline 17 & 0,1272 & 0,1140 & 0,1050 & $0,|34|$ & 0,1083 & 0,1333 \\
\hline 18 & 0,0985 & 0,0782 & 0,0716 & 0,0899 & 0,0708 & 0,1033 \\
\hline Age médian & $|5|$, & 15,2 & 15,2 & 15,2 & 15,2 & 15,3 \\
\hline \multicolumn{7}{|c|}{ Test d'homogénéité des courbes de transition } \\
\hline Test & \multicolumn{2}{|l|}{$\mathrm{Khi}^{2}$} & \multicolumn{2}{|l|}{ DL } & \multicolumn{2}{|l|}{$\operatorname{Pr}>\mathrm{Khi}^{2}$} \\
\hline$-2 \log (L R)$ & \multicolumn{2}{|l|}{27.8031} & \multicolumn{2}{|l|}{6} & \multicolumn{2}{|l|}{0.0001} \\
\hline
\end{tabular}

Note les années de naissance constituent la différence en millésime entre la date de l'enquête et les âges exacts. Source : Enquête démographique et de santé du Sénégal (EDS), 1986. 
Tableau 2: Proportion des femmes non mariées selon l'âge au début de l'intervalle et la génération

\begin{tabular}{|c|c|c|c|c|c|c|}
\hline $\begin{array}{l}\text { Age à } \\
\text { l'enquête }\end{array}$ & $15-19$ & $20-24$ & $25-29$ & $30-34$ & $35-39$ & $40-44$ \\
\hline $\begin{array}{l}\text { Année de } \\
\text { naissance }\end{array}$ & $\begin{array}{l}1966- \\
71\end{array}$ & $196 \mid-66$ & $\begin{array}{l}1957- \\
61\end{array}$ & $\begin{array}{l}1951- \\
57\end{array}$ & $\begin{array}{l}1947- \\
51\end{array}$ & $194 \mid-47$ \\
\hline \multicolumn{7}{|l|}{$\begin{array}{l}\text { Age au début } \\
\text { de l'intervalle }\end{array}$} \\
\hline II & I & I & 0.9988 & I & I & I \\
\hline 12 & 0.9990 & 0.9955 & 0.9916 & I & I & I \\
\hline 13 & 0.9846 & 0.9844 & 0.9809 & 0.9817 & 0.9792 & 0.9833 \\
\hline 14 & 0.9405 & $0.934 I$ & 0.9320 & 0.9284 & 0.9250 & 0.9200 \\
\hline 15 & 0.8677 & 0.8458 & 0.8305 & 0.8308 & 0.8042 & 0.8000 \\
\hline 16 & 0.7175 & 0.6514 & 0.6014 & 0.5640 & 0.527 I & 0.5133 \\
\hline 17 & 0.6203 & 0.5218 & 0.4618 & 0.4253 & 0.3813 & 0.3633 \\
\hline 18 & 0.5330 & 0.4123 & 0.3628 & 0.3247 & 0.2667 & 0.2567 \\
\hline 19 & 0.4887 & 0.3542 & 0.2983 & 0.2470 & 0.1854 & 0.1900 \\
\hline Age médian & 18.74 & 17.20 & 16.73 & 16.46 & 16.19 & 16.09 \\
\hline \multicolumn{7}{|c|}{ Test d'homogénéité des courbes de transition du mariage } \\
\hline Test & \multicolumn{2}{|l|}{$\mathrm{Khi}^{2}$} & \multicolumn{2}{|l|}{$\mathrm{DL}$} & \multicolumn{2}{|c|}{$\mathrm{Pr}>\mathrm{Khi}^{2}$} \\
\hline Log-Rank & \multicolumn{2}{|c|}{343.0535} & \multicolumn{2}{|l|}{6} & \multicolumn{2}{|l|}{0.0001} \\
\hline
\end{tabular}

Source : Enquête démographique et de santé du Sénégal (EDS), 1986. 
Tableau 3: Test d'association entre l'âge à la première union et les variables

\begin{tabular}{|c|c|c|}
\hline & $\begin{array}{l}\text { Test du } \mathrm{Khi}^{2} \\
\text { univarié } \\
\text { Long rank }\end{array}$ & Wilcoxon \\
\hline Variables & t- statistique & t- statistique \\
\hline Survie de la mère & $98.37^{* *}$ & $38.89 * *$ \\
\hline survie du père & $74.13^{* *}$ & $50.83^{* *}$ \\
\hline Age à la puberté & 1694.1 & 1392.2* \\
\hline Résidence & $-290.8^{* * * *}$ & $-192.0 * * *$ \\
\hline Education & $420.8 * * *$ & $264.2 * * * *$ \\
\hline \multicolumn{3}{|c|}{ Prise en compte des interactions entre les variables } \\
\hline Variables & Incrément en Khi² & Incrément en $\mathrm{Khi}^{2}$ \\
\hline Education & $140.8 * * *$ & $277.8^{* * * *}$ \\
\hline Résidence & $31.92 * * *$ & $39.73 * * *$ \\
\hline Age à la puberté & $7.14 * *$ & $7.458^{* * *}$ \\
\hline Survie du père & $5.12 *$ & $4.475^{*}$ \\
\hline Survie de la mère & $2.98 *$ & 2.113 \\
\hline
\end{tabular}

Source : Enquête démographique et de santé du Sénégal (EDS), 1986.

Note : ***, ** ou * signification à $0.01,0.05,0.10$ 
Tableau 4: Proportion des femmes sans première naissance par génération

\begin{tabular}{|c|c|c|c|c|c|c|}
\hline $\begin{array}{l}\text { Age au } \\
\text { début de } \\
\text { l'intervalle }\end{array}$ & $15-19$ & $20-24$ & $25-29$ & $30-34$ & $35-39$ & $40-44$ \\
\hline 12 & - & 0.9989 & 0.9988 & - & - & 0.9933 \\
\hline 13 & 0.9979 & 0.9966 & 0.9964 & 0.9970 & 0.9958 & 0.9800 \\
\hline 14 & 0.9908 & 0.9888 & 0.9773 & 0.9832 & 0.9854 & 0.9300 \\
\hline 15 & 0.9713 & 0.9508 & 0.9403 & 0.9405 & 0.9583 & 0.8333 \\
\hline 16 & 0.9149 & 0.8615 & 0.8600 & 0.8430 & 0.8750 & 0.7067 \\
\hline 17 & 0.8044 & 0.7520 & 0.7279 & 0.7104 & 0.7292 & 0.6067 \\
\hline 18 & 0.6859 & 0.6011 & 0.6110 & 0.6082 & $0.577 \mid$ & 0.4633 \\
\hline 19 & $0.575 I$ & 0.4961 & 0.5048 & 0.4985 & 0.4729 & 0.3867 \\
\hline 20 & - & 0.4056 & 0.3890 & 0.4040 & 0.3875 & \\
\hline $\begin{array}{l}\text { Age } \\
\text { médian }\end{array}$ & - & 18.96 & 19.04 & 18.18 & 18.74 & 18.74 \\
\hline Effectifs & 975 & 895 & 838 & 656 & 480 & 300 \\
\hline
\end{tabular}

Source : Enquête démographique et de santé du Sénégal (EDS), 1986.

Tableau 5: Répartition des femmes de 15-49 ans selon le type de conceptions et de naissances.

\begin{tabular}{||l|l|l||}
\hline Catégories & Effectif & Pourcentage \\
\hline \hline $\begin{array}{l}\text { Conception et naissance } \\
\text { prénuptiales }\end{array}$ & 464 & 14.1 \\
\hline \hline $\begin{array}{l}\text { Conception avant et } \\
\text { naissance après le mariage }\end{array}$ & 414 & 12.5 \\
\hline \hline $\begin{array}{l}\text { Naissance légitime ane à } \\
\text { Naissances de mères } \\
\text { encore célibataires à } \\
\text { l'enquête }\end{array}$ & 2423 & 70.2 \\
\hline \hline \begin{tabular}{l} 
Total \\
\hline \hline
\end{tabular} & 3301 & 100 \\
\hline
\end{tabular}


Source : Enquête démographique et de santé du Sénégal (EDS), 1986.

Tableau 6: Proportions cumulées des conceptions avant le mariage à l'âge de 18 ans selon la génération

\begin{tabular}{|c|c|c|c|}
\hline $\begin{array}{l}\text { Groupes } \\
\text { d'âge }\end{array}$ & $\begin{array}{l}\text { Proportions } \\
\text { cumulées de } \\
\text { conceptions avant le } \\
\text { mariage }\end{array}$ & $\begin{array}{l}\text { Rapport sur les } \\
\text { femmes ayant } \\
\text { vécu } \quad \text { un } \\
\text { événement }(\%)\end{array}$ & Total \\
\hline $15-19$ ans & 149 & 35 & 975 \\
\hline $20-24$ ans & 210 & 32 & 895 \\
\hline $25-29$ ans & 185 & 26 & 838 \\
\hline $30-34$ ans & 194 & 26 & 656 \\
\hline $35-39$ ans & 181 & 21 & 480 \\
\hline $40-44$ ans & 220 & 27 & 300 \\
\hline Ensemble & 1853 & 27 & 4415 \\
\hline
\end{tabular}

Source : Enquête démographique et de santé du Sénégal (EDS), 1986

\section{BIBLIOGRAPHIE}

- Adetoro O. and A. Agah."The Implications of Childbearing in Postpubertal Girls in Sokoto, Nigeria" International Journal of Gyneacological and obstetrics 1988 aug, 27 (I), pp 73-77.

- Akingba j. b.. The problem of unwanted pregnancies in Nigeria Today, New York 197I, II7 p.

- Akuffo F.O."Teenage Pregnancies and School Dropouts:The Relevance of Family Life Education and Vocational Training to Girls Employement Opportunities", in C.Oppong ed.,Sex Roles, Population and Development in West Africa, Heinemann, Portsmouth, N.H., and James Currey, London 1987, pp. 138-I54.

- Aumont Isabelle.Cohabitation et mariage: un essai de mesure démographique d'après l'enquête sur la fécondité au Canada de 1984. Mémoire de Maîtrise, Département de Démographie, Septembre 1987, 164 p.
- Ajayi Ayo, Leah Marangu, Janice Miller and John Paxman. "Adolescent Sexuality and Fertility in Kenya: A Survey of Knowledge, Perceptions and Practices" Studies in Family Planning Vol.22, No. 4, July/August I99I, pp. 205-2I6.

- Bamikale Feyisetan and Anne R. Pebley." Premarital Sexuality in Urban Nigeria", Studies in Family Panning, Vol. 20, No. 6,Novembre/decembre 1989, pp 343-354.

- Buck C. and K. Stavraky. "The Relationship between Age at Menarche and Age at Marriage among Childbearing Women" Human Biology Vol 39 No. 93, 1967.

- Caldwell John, Pat Caldwell, Pat Quiggin."The Social Context of AIDS in Sub-Saharan Africa", Population and Development Review Vol. No. I, 1990, pp 189-234.

- Cherlin Andrew and Nancy Riley. Adolescent Fertility: An Emerging Issue in Sub-Saharan Africa Population 
Health and Nutrition Department, Washington D.C: World Bank, 1986.

- Chui James.Policies and Programs on Adolescent Fertility in Developing Countries: an Integrated Approch New York:United Nations Fund for Population Activities (UNFPA), 1978.

- Demehin Ade Olowo." Sexual Attitudes in Traditional and Modern Yoruba Society", International Quaterly of Community Health Education Vol.4 No. 3, I983, pp 23I-238

- Diop Nafissatou J. "Adolescence et société". Vie et santé Revue du Réseau de Recherche en Santé de la Reproduction en Afrique, No. 8, Juillet 1991, pp. 22-27.

- Diop Nafissatou J. La fécondité des adolescentes au Sénégal. Thèse de doctorat. Département de démographie, Université de Montréal, 1993, 260 pages.

- Elder Glen." Adolescence in Historical Perspectiqes", Handbook of Adolescent Psychology ed. by Joseph Adelson, New York: Wiley, 1980, pp 3-46.

- Gyepi-Garbrah Ben, Douglas Nichols and G.M.K. Kpedekpo. Adolescents Fertility in Sub-Sahara Africa, Boston Massachussets The Pathfinder Fund, 1985e.

- Gyepi-Garbrah Ben. Fertility and Marriage in Adolescents in Africa.African Population Conference/Congrès Africain de Population Dakar Senegal,Novembre 7-12 1988, Belgium, International Union for the scientific study of population, Vol.2, 1988, pp 5.3.1-15

- Hobcraft John. Reproductive Change in Developing Countries, John Cleland and
John Hobcraft, eds. New York: Oxford University Press, 1985.

- Jones Elise, Jacqueline Forrest, Noreen Goldman, Stanley Henshaw, Richard Lincoln, Jeannie Rosoff, Charles Westoff, Deirdre Wulf." Teenage Pregnancy in Industrialised Countries: A Study Sponsored by the Alan Guttmacher Institute, New Haven-Yale University Press, 1986.

- Kalbfleisch, J.D and R.L. Prentice. The Statistical Analysis of Failure Time Data, New-York: John Wiley \& sons, 1980.

- Ladipo O.A., Douglas Nichols, John Paxman, Gorgio Delano, S.E.Kelly, E.O.Otolorin." Sexual Behavior, Contraceptive Practice and Reproductive Health among the Young Unmarried Population in Ibadan, Nigeria, Final Report, Family Health International, Research Triangle Park, North Carolina, 1983

- Liskin Laurie."Jeunesses des années 80: problèmes sociaux et sanitaires", Population Reports Sujets spéciaux, série M, No. 9, Jan/Fev 1987.

- LeBourdais Celine et Hélène Desrosiers."Les femmes et l'emploi: une analyse de la discontinuité des trajectoires féminines" Recherche féministes Vol.3 No. I, 1990, pp.I I9-134

- Léridon Henri. "Aspects biométriques de la fécondité humaine" Travaux et Documents cahier de l'INED n 65, 1973.

- Lesthaeghe Ron. " Social Organisation, Economic Crisis and the Future of Fertility Control in Africa" in Reproduction and Social Organisation 
in Sub-Saharan Africa, ed. R. Lesthaeghe, Berkeley: University of California Press, 1988.

- Ndiaye Salif, Sarr Ibrahima, Ayad Mohamed. Enquête Démographique et de Santé au Sénégal 1986, Division des Enquêtes et de la Démographie et Institute for Resource Development/Westinghouse, Dakar, Mars 1988.

- Nichols Douglas, O. Ladipo, John Paxman, E. Octolorin." Sexual Behaviour, Contraceptive Practice and Reproductive Health among Nigerian Adolescents", Studies in Family Planning Vol. I7, No. 2, Mar/Apr 1986, pp 100-106.

- Njogu W.. Social Background of Teenage Mothers. Research Report Paper Presented at the IPPF Seminar on Adolescent Fertility Managment, Nairobi, Oct 1980.

- Omu A., A. Oronsaye, M. Faal, E. Asuquo." Adolescent Induces Abortion in Benin City, Nigeria", International Journal of Gyneacology and Obstetric Vol. 19 No. 6,Decembre 198I, pp 495-499.

- Oronsaye A., G. Odiasse." Attitudes Toward Abortion and Contraception among Nigerian Secondary Schoolgirls" International Journal of Gyneacology and Obstetrics Vol.2I, No. 5, October 1983 pp 423-426.

- Pressat Roland, 1983.L'analyse démographique quatrième édition, Presses Universitaires de France.

- Presser H. "Age at Menarche, SocioSexual Behavior and Fertility. Social Biology Vol. 25, 1978, pp 94.
- Senderowitz Judith and John Paxman." Adolescent Fertility: Worldwide Concerns", Population Bulletin Vol.40, No. 2, April I985,

- Smith David P."Life Table analysis" WFS Technical Bulletins No. 6, 1980, 25 p.

- Tietze Christopher, S.K. Henshaw. "Induced Abortion: A World Review, ed. New York, Population Council, 1986.

- Torres Aida." Does Your Mother Know...?" Family Planning Perspectives Vol.I0, No. 5, Septembre/Octobre 1978, pp 280-282.

- United Nations .The Situation of Youth in the 1980s and Prospects and Challenges for the Year 2000, New York, 1986.

- United Nations.Adolescent Reproductive Behaviour:Evidence from Developing Countries Vol.2, Population Studies No. I09/add I, New York, I989a.

- Westoff Charles, Gerard Calot, Andrew Foster." Teenage Fertility in Developed Nations", Family Planning Perspectives Vol.15, No. 3, May/June 1983, pp I05-II0.

- Westoff Charles."Unintended Pregnancy in America and Abroad" Family Planning Perspectives Vol.20 $\mathrm{n}$ 6, November/December 1988, pp 254-26I.

- World Health Organisation. Young People Health-a Challenge for Society, Technical Report Series 73I, Geneva, 1986.

- Zeidenstein Georges."Adolescent Fertility And The Health And Status of Women, Bulletin, Vol 6, No. 10, October 1989, $5 \mathrm{p}$. 
- Zelnik Melvin, John Kantner,

Kathleen Ford. Sex and Pregnancy in

Adolescence. Sage Library of Social

Research, Vol.133, Beverly Hills,

California: Sage Publications, 198I. 